\title{
Mesenchymal stem cells alleviate liver injury induced by chronic-binge ethanol feeding in mice via release of TSG6 and suppression of STAT3 activation
}

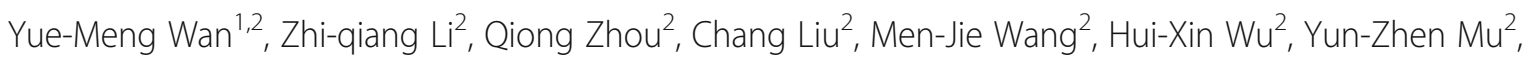
Yue-Feng $\mathrm{He}^{2}$, Yuan Zhang ${ }^{3}$, Xi-Nan $\mathrm{Wu}^{2^{*}}$, Yu-Hua $\mathrm{Li}^{1}$, Zhi-Yuan $\mathrm{Xu}^{1}$, Hua-Mei Wu${ }^{1}$, Ying $\mathrm{Xu}^{1}$, Jin-Hui Yang ${ }^{1}$ and Xiao-Fang Wang ${ }^{4}$

\begin{abstract}
Background: Mesenchymal stem cells (MSCs) are a population of pluripotent cells that might be used for treatment of liver disease. However, the efficacy of MSCs for mice with alcoholic hepatitis (AH) and its underlying mechanism remains unclear.

Methods: MSCs were isolated from the bone marrow (BM) of 4-6-week-old male C57BL/6 N mice. AH was induced in female mice by chronic-binge ethanol feeding for 10 days. The mice were given intraperitoneal injections of MSCs with or without transfection or AG490, recombinant mouse tumor necrosis factor (TNF)-a-stimulated gene/ protein 6 (rmTSG-6), or saline at day 10. Blood samples and hepatic tissues were collected at day 11. Various assays such as biochemistry, histology, and flow cytometry were performed.

Results: MSCs reduced AH in mice, decreasing liver/body weight ratio, liver injury, blood and hepatic lipids, malondialdehyde, interleukin (IL)-6, and TNF-a, but increasing glutathione, IL-10, and TSG-6, compared to control mice. Few MSCs engrafted into the inflamed liver. Knockdown of TSG-6 in MSCs significantly attenuated their effects, and injection of rmTSG- 6 achieved similar effects to MSCs. The signal transducer and activator of transcription 3 (STAT3) was activated in mice with AH, and MSCs and rmTSG-6 inhibited the STAT3 activation. Injection of MSCs plus AG490 obtained more alleviation of liver injury than MSCs alone.
\end{abstract}

Conclusions: BM-MSCs injected into mice with AH do not engraft the liver, but they secrete TSG-6 to reduce liver injury and to inhibit STAT3 activation.

Keywords: Alcoholic hepatitis, Bone marrow mesenchymal stem cells, Oxidative stress, Neutrophil infiltration, Macrophage infiltration, Cytokines, TSG-6, STAT3 activation

\section{Introduction}

Alcoholic liver disease (ALD) is a leading cause of chronic liver disease worldwide with high morbidity and mortality [1]. According to a recent review, the prevalence of ALD in China is about $4.5 \%$, which, quite alarmingly, is about to overtake the USA (6.2\%) and European countries (6\%), and has already dwarfed neighboring Japan (1.56-2.34\%) [2].

\footnotetext{
*Correspondence: wxnprof2011@163.com

${ }^{2}$ Public Health Institute of Kunming Medical University, Kunming City 650500,

Yunnan Province, China

Full list of author information is available at the end of the article
}

ALD is a complex disease that encompasses a wide spectrum of hepatic lesions, including steatosis, alcoholic hepatitis $(\mathrm{AH})$, progressive fibrosis, cirrhosis, and superimposed hepatocellular carcinoma, which may occur separately, simultaneously, or sequentially in a patient. $\mathrm{AH}$ is a necroinflammatory process that may cause cirrhosis in $40 \%$ of cases due to its association with the fastest progression of fibrosis, and patients without $\mathrm{AH}$ are at low risk of developing

(c) The Author(s). 2020 Open Access This article is distributed under the terms of the Creative Commons Attribution 4.0 International License (http://creativecommons.org/licenses/by/4.0/), which permits unrestricted use, distribution, and 
cirrhosis [3, 4]. Severe form of AH is associated with very high short-term mortality, about $20-50 \%$ at 3 months, and represents one of the most deadly diseases [5]. Despite cellular injury, oxidative stress, inflammation, and bacterial translocation are known driving factors for ALD, and multiple attempts have been made to improve patient outcomes, there is still no more successful treatment for this illness than alcohol abstinence and corticosteroid exposure to those with severe $\mathrm{AH}$. However, these treatments are not successful in about $40 \%$ of cases [6]. Therefore, there is an urgent need to explore other new and effective therapies for this illness.

Mesenchymal stem cells (MSCs) are a population of pluripotent cells that can be obtained from many body sites, such as the bone marrow (BM) [7, 8], adipose tissue $[9,10]$, umbilical cord $[11,12]$, and placenta [13]. These cells are easy to isolate, can rapidly expand in culture and differentiate into multiple lineages of cells, and seem to be an ideal source for cytotherapy. Previous studies have reported their efficacy in treating myocardial infarction, inflammatory bowel disease, pancreatitis, burn-induced excessive inflammation, liver failure, corneal injury, and peritonitis [7-15]. The underlying mechanism for this therapy was mainly attributable to the tumor necrosis factor (TNF)-a-stimulated gene/protein 6 (TSG-6) secreted by MSCs after injection and activation [7-11, 14, 15]. However, in the literature, there is still very scarce data about the efficacy of MSCs for ALD and its underlying mechanisms up to date.

Previous studies showed that TSG-6 was able to inhibit many signaling pathways, such as the mitogen-activated protein kinase (MAPKs) pathway [11] and nuclear factor (NF)- $\mathrm{kB}$ pathway [15], and these signaling pathways had also been extensively studied in ALD [16, 17]. Previous studies also reported that the signal transducer and activator of transcription 3 (STAT3) signaling pathway was implicated in the pathogenesis of ALD [18], and acute ethanol intake could activate STAT3 signaling [19, 20]. However, it remains unclear how STAT3 signaling is related to AH and whether MSCs can exert their efficacy in AH through TSG-6 and/or interaction with STAT3 signaling.

Therefore, we conducted the present study to investigate the following three issues: first, to confirm the efficacy of MSCs for AH; second, to explore the association of TSG-6 with the efficacy of MSCs for AH; third, to investigate the relevance of STAT3 signaling to the treatment of AH by MSCs.

\section{Materials and methods}

\section{Mice}

C57BL/6 N mice were purchased from the Experimental Animal Center of Kunming Medical University. All 8- 10-week-old female mice were used unless specified. All animal experiments were approved by the Ethics Committees of Kunming Medical University (No. KMMU 2019067) and were conducted according to the Guideline of Animal Care and Use Committee of Kunming Medical University.

Isolation, characterization, and transfection of BM-MSCs MSCs were isolated from the $\mathrm{BM}$ of the tibias and femurs of 4-6-week-old male C57BL/6 $\mathrm{N}$ mice as previously described $[7,8]$. The isolation, culture, characterization, and transfection of MSCs were described in Additional file 1, Additional file 2, Additional file 3, and Additional file 4. MSCs at passages 2-3 were used in the subsequent experiments.

\section{Model of $\mathrm{AH}$ and experimental groups}

$\mathrm{AH}$ was generated by using the Lieber-DeCarli liquid diet plus alcohol gavage according to the National Institute on Alcohol Abuse and Alcoholism (NIAAA) protocol [18], with slight modification aiming to induce more severe $\mathrm{AH}$ by introducing two more doses of ethanol gavage. The diets were obtained from TROPHIC Animal Feed High-Tech Co. Ltd. (Hai'an, Jiangsu, China) and were prepared fresh daily. All C57BL/6N mice were fed a nutritionally adequate liquid control diet for 5 days (acclimatization period), then divided into the following seven groups: control, $\mathrm{AH}, \mathrm{AH}+\mathrm{MSCs}(\mathrm{AH}$ transplanted with normal MSCs), AH+sc-MSCs (AH transplanted with MSCs transfected by Lentiscrambled-siRNA), AH+siTSG-6-MSCs (AH transplanted with MSCs transfected by Lenti-TSG-6siRNA), AH+recombinant mouse (rm) TSG-6 (AH followed by rmTSG-6), and AH+MSCs+AG490 (AH transplanted with normal MSCs plus AG490 injection). The AH groups were fed a liquid ethanol diet containing $5 \%$ ethanol $(\mathrm{v} / \mathrm{v})$ for 10 days, while the control group was pair-fed control diet for 10 days (modeling period).

\section{Experimental treatment}

At days 3, 6, and 11 of the modeling period, mice in the $\mathrm{AH}$ groups were gavaged a single dose of ethanol (31.5\% ethanol(v/v), $400 \mu \mathrm{l} /$ mouse), while mice in the control group were gavaged isocaloric dextrin maltose ( $45 \%(\mathrm{w} / \mathrm{v}), 400 \mu \mathrm{l} / \mathrm{mouse})$. At day 10 , MSCs with or without transfection were intraperitoneally (i.p.) administered to the AH+MSCs, AH+sc-MSCs, AH+siTSG-6MSCs, and AH+MSCs+AG490 groups $\left(5 \times 10^{6}\right.$ cells/ mouse); following injection of MSCs, an additional dose of AG490 (Tocris, Bristol, UK) was given to the $\mathrm{AH}+\mathrm{MSCs}+\mathrm{AG} 490$ group $(20 \mu \mathrm{g} / \mathrm{mouse}$, i.p.); rmTSG-6 (R\&D, Minneapolis, MN, USA) was injected to the 
AH+rmTSG-6 group (10 $\mu \mathrm{g} /$ mouse, i.p.); equal volumes of saline were administered to the control or AH group per protocol requirement. The gavage was always performed in the morning, and mice were then maintained on control or ethanol diet. After ethanol gavage, mice were lethargic and tachypneic, but they recovered within 4-6 h. The mice were always anesthetized $9 \mathrm{~h}$ post the last gavage with samples of blood, peritoneal fluid lavage, and liver tissues harvested for further analyses (Additional file 1).

\section{Other reagents and methods}

Other assays are described in Additional files 1 and 9, including blood chemistry, liver histology and immunohistochemistry, flow cytometry, myeloperoxidase (MPO) activity assay, enzyme-linked immunosorbent assay (ELISA), reverse transcription quantitative PCR (RTqPCR) and Western-blot assays, and hepatic oxidative stress analysis.

\section{Statistical analysis}

Data are expressed as means \pm standard deviation (SD) and were analyzed using GraphPad PRISM software, version 8.02 (GraphPad Software Inc., La Jolla, CA, USA). A Student's $t$ test (for two group comparisons) or a Kruskal-Wallis one-way ANOVA followed by Tukey's post hoc test (for three or more group comparisons) was used for statistical analyses. A value of $P<0.05$ was considered statistically significant.

\section{Results}

Intraperitoneal injection of MSCs ameliorated ethanolinduced liver injury

Until day 11, similar body weight gains were observed among all groups, though liver/body weight ratio was higher in the $\mathrm{AH}$ group compared to the control group (Additional file 2: Figure S1j, k). i.p. injection of MSCs $\left(5 \times 10^{6}\right.$ cells/mouse) significantly improved the liver injury parameters such as liver/body weight ratio (Additional file 2: Figure S1k,l), serum alanine aminotransferase (ALT) (Fig. 1a), aspartate aminotransferase (AST) (Fig. 1b), total triglyceride (TG), total cholesterol (TC) levels (Fig. 1c, d), hepatic TG and TC concentrations (Fig. 1e, f), hepatic malondialdehyde (MDA) and glutathione (GSH) (Fig. 1g, h), hepatic steatosis, hepatocyte ballooning, necroinflammation, and corresponding histology scores (Fig. 1i-o) as well as hepatic infiltration by neutrophils (Additional file 5: Figure S4) and monocytes/macrophages (Additional file 6: Figure S6). No significant signs of liver injury except hepatocyte ballooning were detected in the control group (Fig. 1i, n). Consistent with these data, we observed that i.p. injection of MSCs $\left(5 \times 10^{6}\right.$ cells/mouse) markedly dampened the systemic and hepatic inflammatory responses as reflected by reduced proinflammatory cytokines (i.e., interleukin (IL)-6, TNF- $\alpha$, cyclo-oxygenase (Cox)-2) (Fig. 2a-h) and elevated anti-inflammatory cytokines (i.e., IL-10, TSG-6) (Fig. 2i-n) in the AH+MSC group as compared to the $\mathrm{AH}$ group.

\section{Low frequency of MSCs engrafted into the inflamed liver}

To evaluate the migratory ability of i.p. injected MSCs, we analyzed the hepatic expression of SRY protein by immunofluorescent assay, since SRY was a male sex determinant gene and its expression in female tissue indicated the presence of allogenic cells. Twenty-four hours after transplantation of MSCs derived from male mice, SRY protein could be sparsely seen in the liver samples of female mice in the AH+MSCs group (Additional file 7: Figure S6a). In control mice, no SRY protein expression could be detected. Moreover, SRY protein expression could be hardly detected in the liver of mice in the AH+MSCs-sc or AH+siTSG-6-MSCs groups that were transplanted with MSCs transfected by lentivirus (Additional file 7: Figure S6b, c). All these data demonstrated that low frequency of MSCs engrafted into the inflamed liver and the therapeutic efficacy of MSCs was unlikely due to the engraftment of MSCs into the liver, but possibly due to certain paracrine factors.

\section{Intraperitoneal injection of MSCs attenuated ethanol- induced liver injury through TSG-6}

We next examined the mechanism by which MSCs exerted their therapeutic effects in AH. Since numerous studies reported that MSCs acted through secreting TSG-6 in other disease models $[7-11,14,15]$, we attempted to investigate whether MSCs also worked via secreting TSG- 6 by the strategy of TSG- 6 knockdown and mimic. Compared to the untreated $\mathrm{AH}$ mice, i.p. injection of $5 \times 10^{6}$ cells/mouse caused a significant reduction of liver/body weight ratio (Additional file 2: Figure S1k,l), liver enzymes (ALT, AST; Fig. 3a, b), and blood and hepatic lipids (TG, TC; Fig. 3c-f). However, these effects were markedly weakened after i.p. injection of $5 \times 10^{6}$ siTSG-6-MSCs/mouse, but were not affected after i.p. injection of $5 \times 10^{6}$ sc-MSCs/mouse. Meanwhile, i.p. administration of rmTSG-6 (10 $\mu \mathrm{g} / \mathrm{mouse})$ showed comparable effects to that of MSCs or sc-MSCs injection (Fig. 3a-f; Additional file 2: Figure S1 l). Oxidative stress is a known driving factor for ALD. We observed that i.p. injection of $5 \times 10^{6} \mathrm{MSCs} /$ mouse markedly lowered the hepatic MDA but increased the hepatic reserve of GSH in comparison to the untreated mice, which was significantly negated by injection of siTSG-6-MSCs but not by sc-MSCs, and administration of rmTSG-6 mimicked the effects of MSCs or sc-MSCs (Fig. 3g, h). Similarly, i.p. injection of $5 \times 10^{6}$ MSCs/ mouse prominently alleviated the hepatic steatosis, 

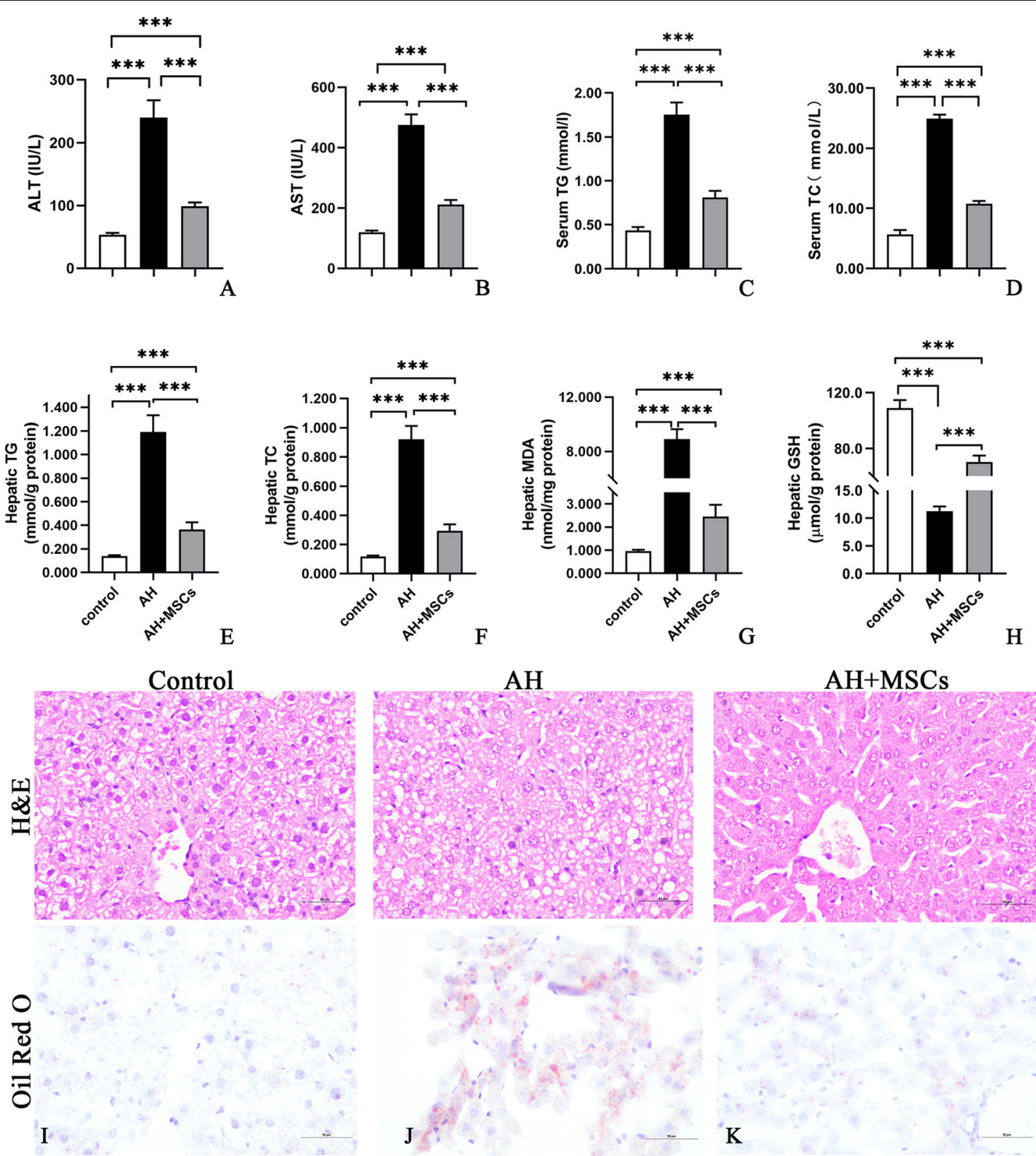

$\mathrm{AH}+\mathrm{MSCs}$
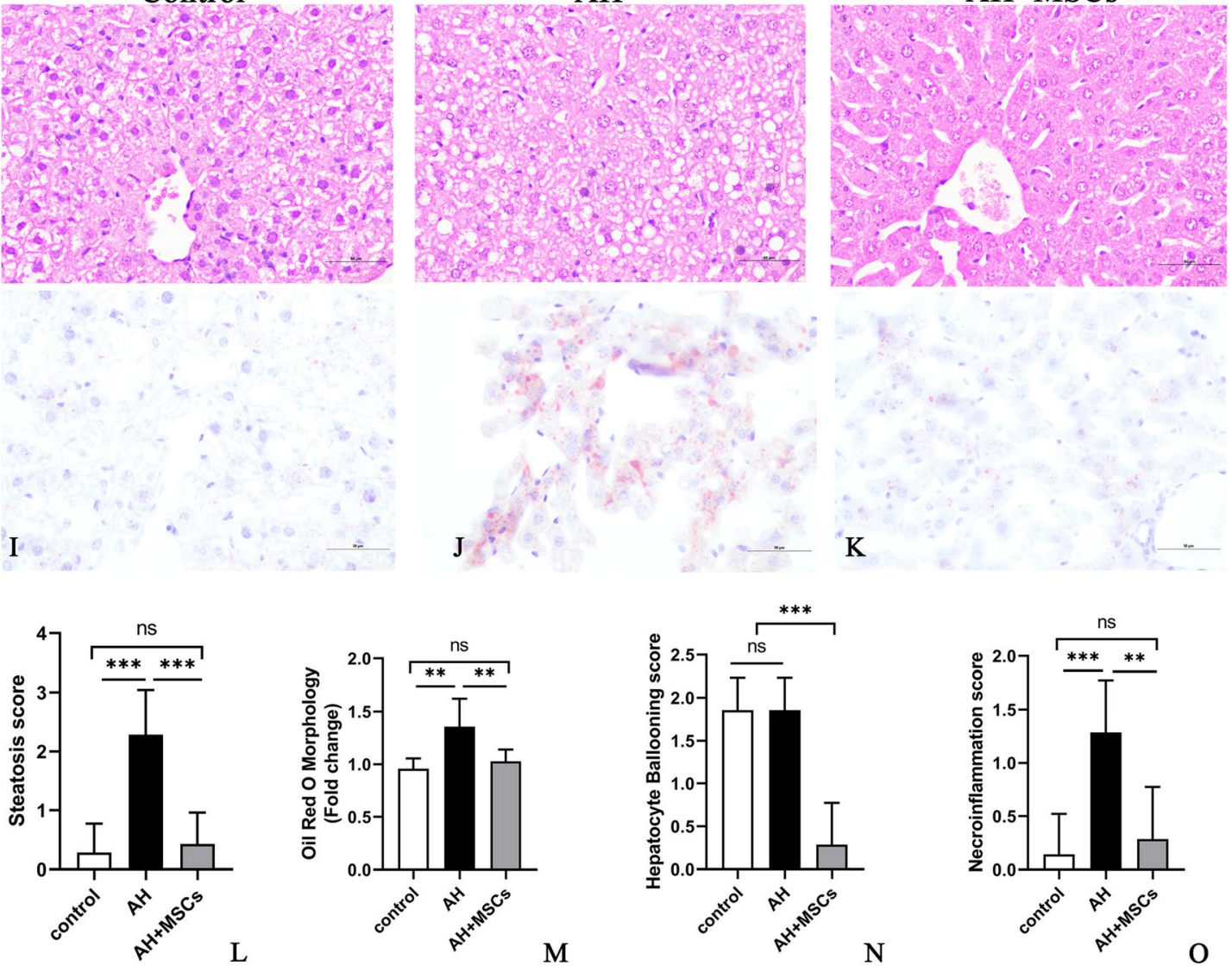

Fig. 1 a-o MSCs ameliorated ethanol-induced liver injury $(\mathbf{a}, \mathbf{b})$, lipid dysregulation (c- $\mathbf{f})$, oxidative stress $(\mathbf{g}, \mathbf{h})$, hepatic steatosis, hepatocyte ballooning, and necroinflammation (i-o). Bars $50 \mu \mathrm{m}$, seven mice per group were used. ${ }^{*} P<0.05$, ${ }^{* *} P<0.01$, ${ }^{* * *} P<0.001$ 


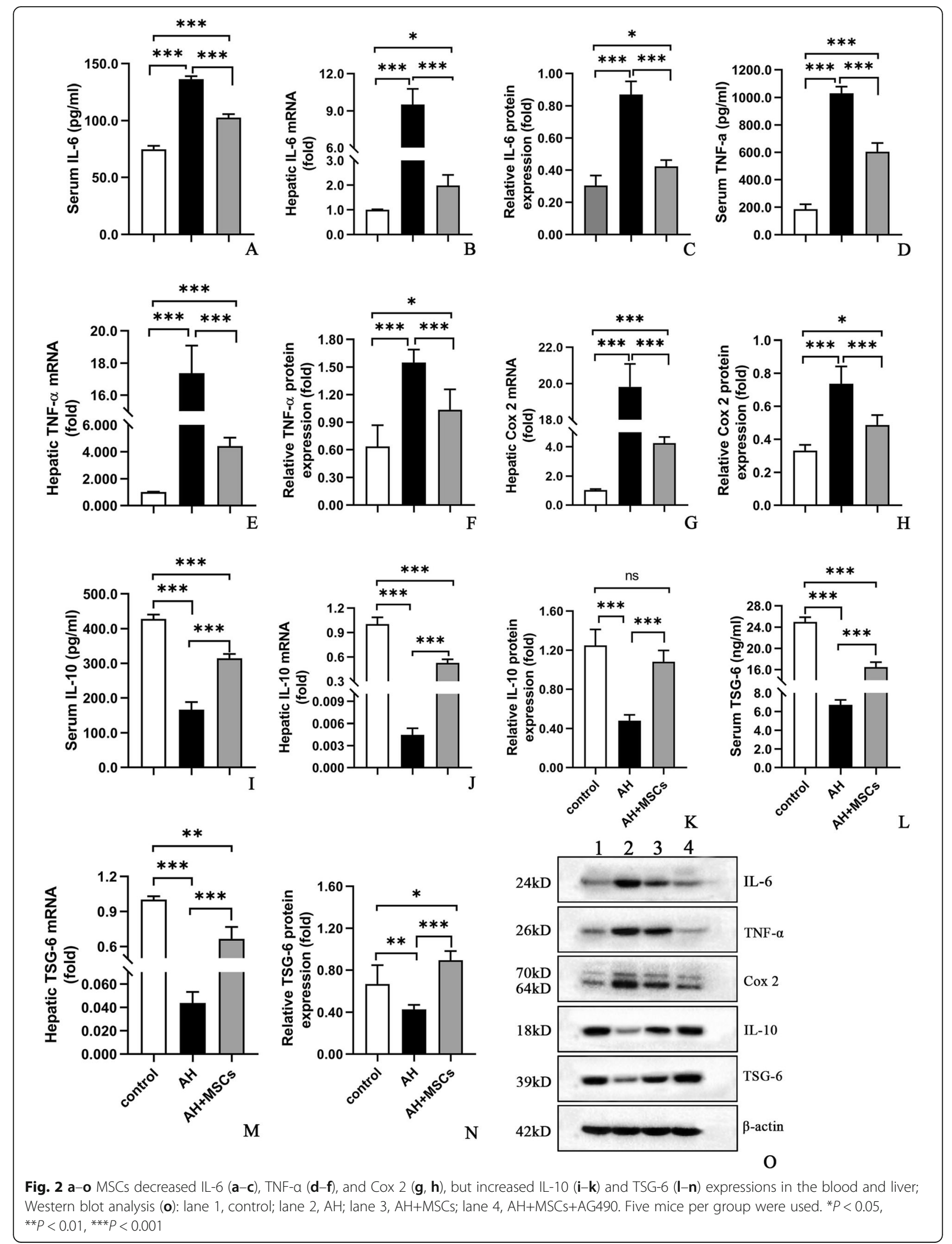






hepatocyte ballooning, necroinflammation and corresponding histology scores (Fig. 4a-i), and infiltration by neutrophils (Additional file 5: Figure S4) and monocytes/macrophages (Additional file 6: Figure S5), which were obviously abolished by injection of siTSG-6-MSCs but not sc-MSCs, and infusion of rmTSG-6 replicated the effects of MSCs or sc-MSCs. In line with these findings, siTSG-6-MSCs but not sc-MSCs markedly decreased the IL-6, TNF- $\alpha$, and Cox 2 levels (Fig. 5a-h) and enhanced the IL-10 and TSG-6 (Fig. 5i-n) levels in the mouse blood and inflamed liver. And exogenous administration of rmTSG-6 produced similar results to those of MSCs and sc-MSCs, but not to siTSG6-MSCs (Fig. 5a-n).

\section{MSCs reduced ethanol-induced liver injury through secreting TSG-6 to inhibit STAT3 signaling}

To investigate the potential mechanism underlying the effects of MSCs on AH in mice, we evaluated the STAT3 signaling, since STAT3 was reported to play a significant role in ALD [18-20]. We observed that STAT3 signaling was significantly activated in the AH group compared to the normal control group, as reflected by increased phosphorylated STAT3 (p-STAT3) level, though the STAT3 level was not altered (Fig. 6a, c, e). After i.p. administration of MSCs or sc-MSCs but not
siTSG-6-MSCs $\left(5 \times 10^{6}\right.$ cells/mouse), p-STATs level was significantly reduced, but STAT3 level was unchanged in comparison to the untreated mice (Fig. 6b, d, f). Moreover, i.p. injection of rmTSG-6 produced similar suppression of p-STAT3 to that of MSCs or sc-MSCs, while STAT3 was not affected (Fig. 6b, d, f). Interestingly, i.p. infusion of AG490 (a STAT3 signaling inhibitor) plus MSCs further suppressed p-STAT3 but not STAT3 level in comparison to MSC administration alone (Fig. 6a, c, e), which also resulted in much more pronounced improvement of $\mathrm{AH}$ (Additional file 8: Figure S7). All these data supported that MSCs acted through TSG-6 that could in turn inhibit the STAT3 signaling.

\section{Discussion}

In the present study, five important findings were presented here. First, we successfully modified the NIAAA model (i.e., chronic-binge model) and induced more severe form of ALD by introducing three ethanol gavages at days 3,6 , and 11 of the modeling period. And the peak ALT and AST levels in our study were $279 \mathrm{IU} /$ $\mathrm{L}$ and $515 \mathrm{IU} / \mathrm{L}$, respectively, as opposed to $250 \mathrm{IU} / \mathrm{L}$ ALT and $420 \mathrm{IU} / \mathrm{L}$ AST in the NIAAA model [18]. Second, we demonstrated that i.p. transplantation of MSCs was effective in treating AH in mice. Third, we provided evidence that MSCs exerted their efficacy in 


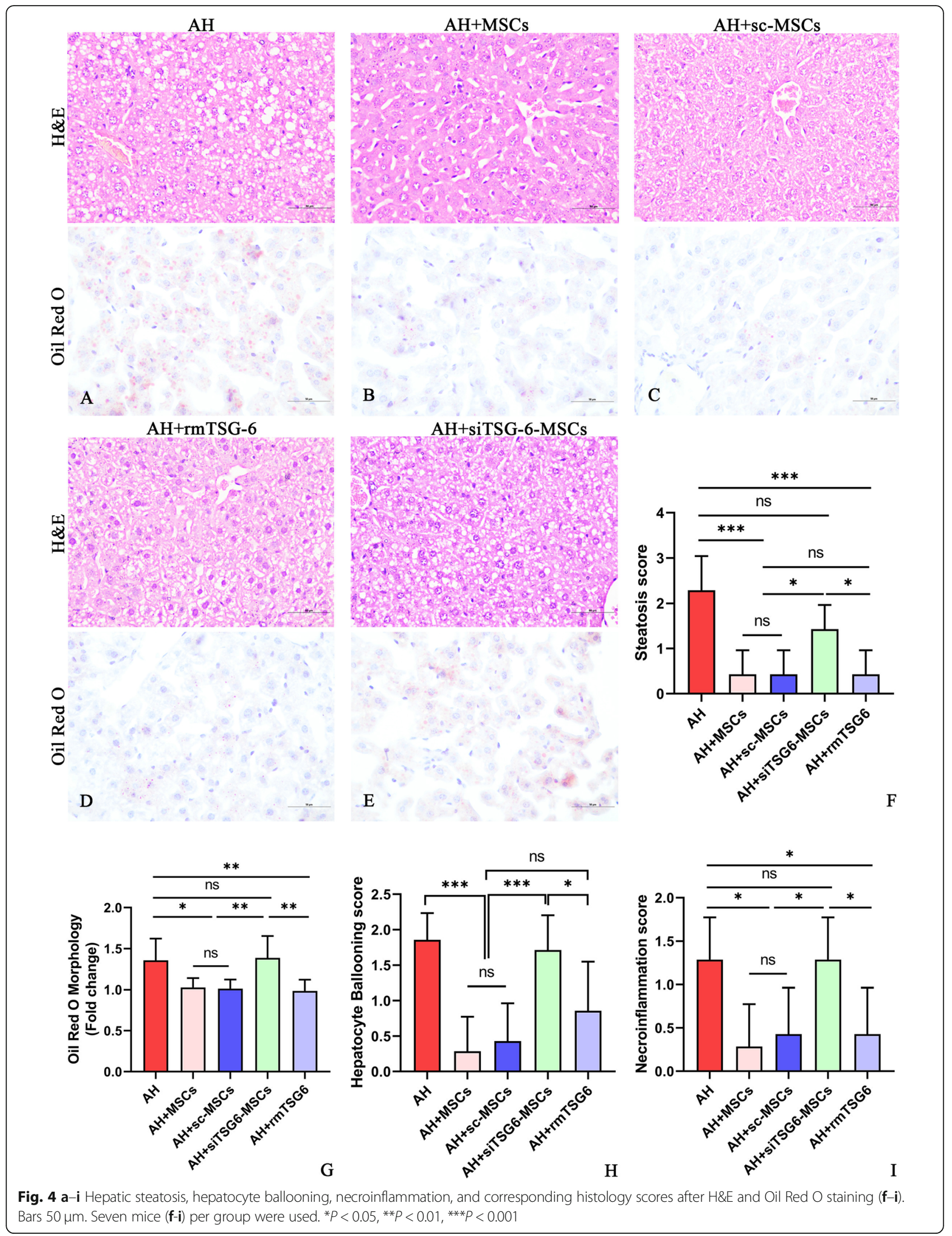




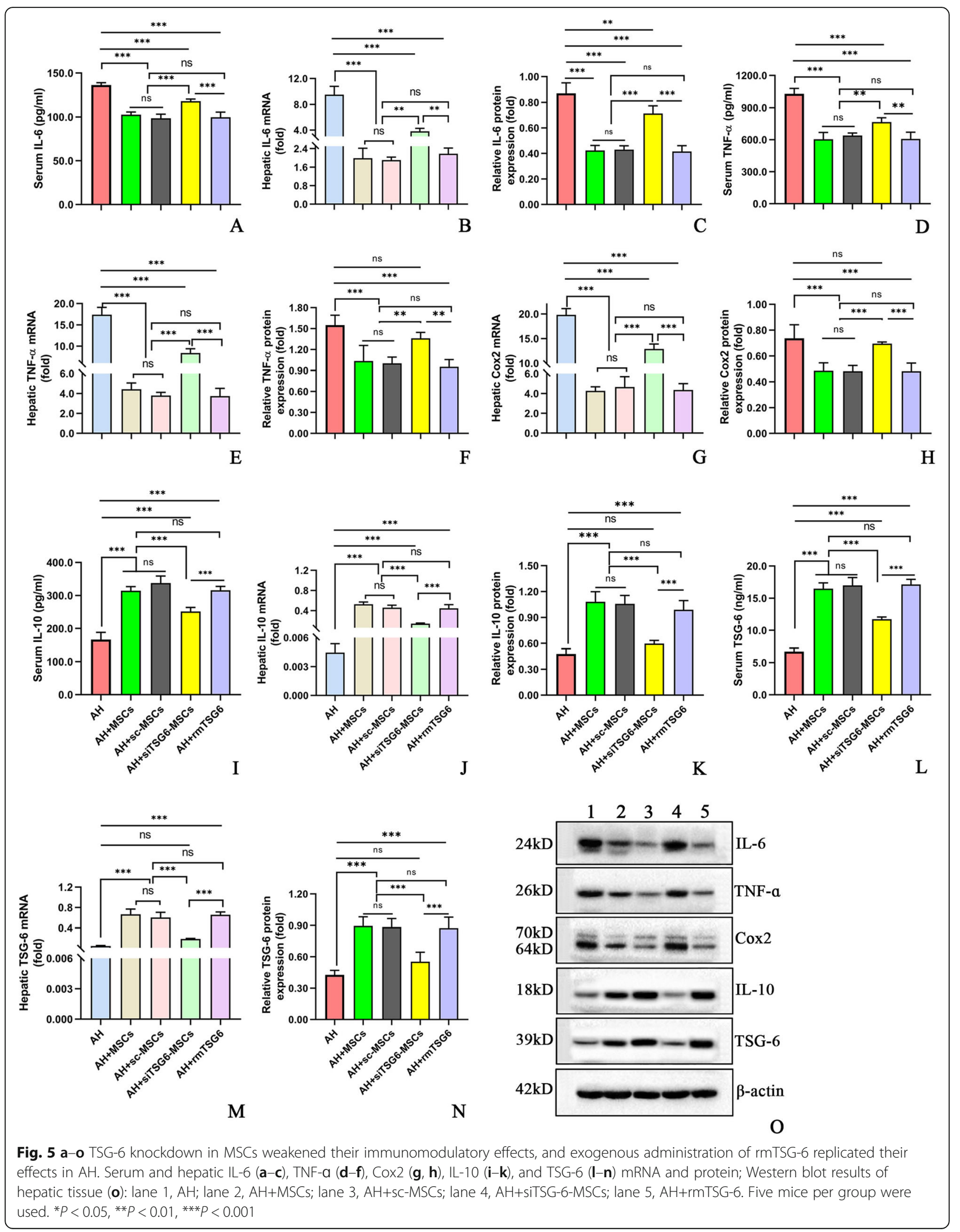



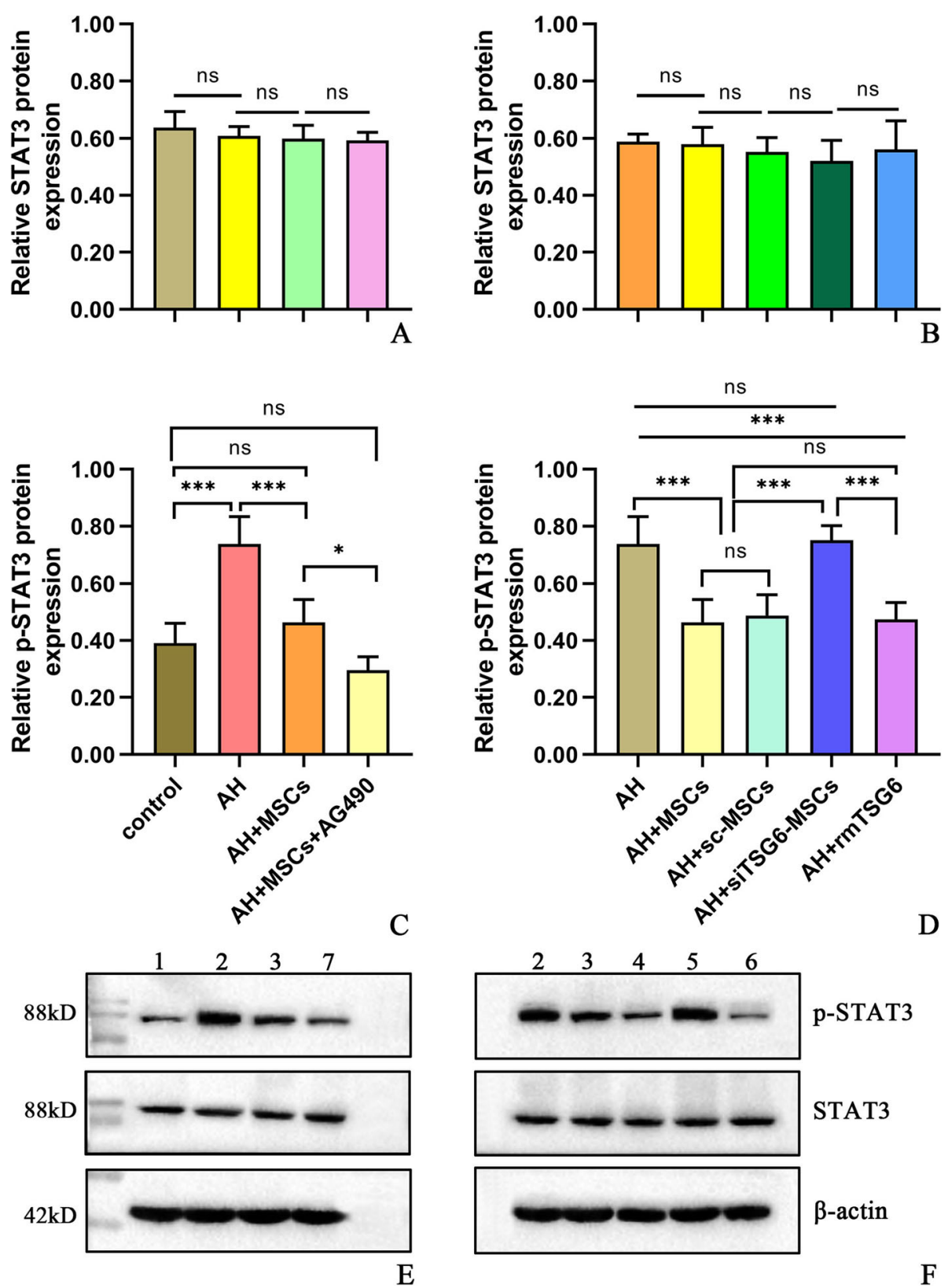

Fig. 6 a-f Hepatic STAT3 (a, b), p-STAT3 levels (c, d), and Western blot results (e, f): lane 1, control; lane 2, AH; lane 3, AH+MSCs; lane 4, AH+sCMSCs; lane 5, AH+siTSG-6-MSCs; lane 6, AH+rmTSG-6; lane 7, AH+MSCs+AG490. Five mice per group were used. ${ }^{*} P<0.05,{ }^{*} P<0.01,{ }^{* * *} P<0.001$

treating AH through secretion of TSG-6. Fourth, we showed that STAT3 signaling was activated in AH, and MSCs could inhibit STAT3 signaling through TSG-6. Fifth, our results suggested that further inhibition of STAT3 signaling by AG490 could enhance the efficacy of MSCs in AH.

Currently, the NIAAA model for ALD has been widely used [18, 21], so we adopted this model to study the efficacy of MSCs. In this study, we observed that i.p. transplantation of MSCs alleviated ethanol-induced $\mathrm{AH}$, and the ALT and AST levels were significantly reduced in mice receiving MSC transplantation compared to the untreated ones (Fig. 1a, b), showing a hepatoprotective effect. Previous studies reported that the blood and hepatic TG and TC levels were elevated in AH mice $[18,22]$. Consistent with these studies, the serum and hepatic TG and TC were significantly higher in the AH group than in the control group, which were markedly reduced after injection of MSCs (Fig. 1c-f). In addition, the H\&E and Oil Red $\mathrm{O}$ staining also illustrated obvious improvement of hepatic steatosis after MSC therapy (Fig. 1i$\mathrm{m})$, exhibiting an anti-steatogenic effect. Oxidative 
stress plays an important role in the pathogenesis of ALD, and many studies reported that oxidative stress and production of reactive oxygen species could induce steatosis, inflammatory cell infiltration, hepatomegaly, fibrosis, and cirrhosis $[18,23,24]$. In agreement with these studies, we noted that MDA, a product of oxidative stress and lipid peroxidation, was much higher, whereas GSH, an antioxidant, was much lower in the $\mathrm{AH}$ group than in the control group (Fig. 1g, h). However, MSC therapy decreased the MDA level but increased the GSH level (Fig. 1g, h), displaying an antioxidative effect.

Previous studies showed that recruitment and activation of neutrophils and macrophages played a significant role in the development of ethanol-induced liver injury $[18,22]$. In concert with these studies, the hepatic numbers of neutrophils and monocytes/macrophages were much greater in the $\mathrm{AH}$ group than in the control group, which were prominently reduced after MSC therapy (Additional files 5 and 6: Figure S4, 5), illustrating an anti-inflammatory effect. An increase of proinflammatory cytokines like TNF-a, IL-1, and IL-6 in the blood and hepatic tissues is a key feature of $\operatorname{ALD}[18,22,25]$. In conformity to these studies $[18,22,25]$, the TNF-a and IL-6 levels were significantly higher in the AH group than in the control group (Fig. 1a-f). As for antiinflammatory cytokines, our study showed that the serum and hepatic IL-10 levels were decreased in the AH group when compared to the control group (Fig. 1ik), which was at odds with a previous study that reported an increase rather than decrease of serum IL10 and hepatic IL-10 mRNA in AH mice [22]. Nonetheless, the serum IL-10 level in the previous study was less than $50 \mathrm{pg} / \mathrm{ml}$ in contrast to about $300 \mathrm{pg} / \mathrm{ml}$ in our study, which may be explained by the different animal modeling process between our study and the previous one (three ethanol gavage vs. one ethanol gavage) that may lead to different severity or stage of AH. Moreover, our study also demonstrated a similar trend of TSG- 6 to IL-10 in both the serum and hepatic levels (Fig. 1l-n). After therapy with MSCs, the proinflammatory cytokines (i.e., TNF- $\alpha$, IL-6) were diminished, whereas the antiinflammatory cytokines (IL-10, TSG-6) were enhanced, showcasing an immunomodulatory effect.

The above findings provided strong evidences that $\mathrm{BM}-\mathrm{MSCs}$ were effective in treating $\mathrm{AH}$. We next sought to explore the underlying mechanism from pro and con two aspects by TSG-6 knockdown and exogenous injection. After knockdown of TSG-6 using TSG-6 siRNA, the efficacy of MSCs was significantly decreased; in contrast, knockdown of TSG-6 using scrambled siRNA as a strict control did not affect the efficacy of MSCs; and exogenous administration of rmTSG-6 achieved comparable effects to MSC therapy (Figs. 3, 4, and 5). These comprehensive data proved robustly that MSCs exerted their efficacy in alleviating $\mathrm{AH}$ in mice via secreting TSG-6, which was in line with the previous studies [7-11, 14, 15].

Previous studies demonstrated that STAT3 signaling played a very important role in liver injury, hepatic inflammation, steatosis, regeneration, and neutrophil trafficking [26-28], and acute ethanol intake could activate STAT3 signaling in monocytes $[19,20]$. Therefore, we hypothesized that hepatic STAT3 signaling was closely related to liver injury, steatosis and inflammation, and hepatic neutrophil and monocyte/macrophage infiltration in AH, and hepatic STAT3 signaling might be a therapeutic target of MSC treatment. However, to our best knowledge, there is no study investigating the effects of MSCs or TSG-6 on hepatic STAT3 signaling in ALD. Consistent with previous studies [19, 20], our study found that hepatic STAT3 signaling was prominently activated in $\mathrm{AH}$, as the p-STAT3 level was much higher in the $\mathrm{AH}$ mice than in the control mice (Fig. 6c, e). And MSC therapy significantly inhibited the hepatic STAT3 signaling, as the p-STAT3 level in mice receiving MSC therapy was markedly decreased compared to the untreated mice, although the STAT3 level was not changed (Fig. 6a, c, e). Using the strategy of TSG-6 knockdown in MSCs and exogenous administration of TSG-6, our data suggested that the inhibition of MSCs on hepatic STAT3 signaling was mainly mediated by TSG- 6 , and TSG- 6 could directly suppress STAT3 signaling (Fig. 6b, d, f). Many cytokines and growth factors can activate STAT3 signaling, including IL-6, IL-10, and IL-22. However, STAT3 activation by different cytokines may elicit opposing effects. For example, STAT3 activation by IL- 6 potentiates proinflammatory responses in peritoneal macrophages [29], whereas STAT3 activation by IL-10 dampens lipopolysaccharide (LPS)-induced inflammatory responses in Kupffer cells [30, 31], and STAT3 activation by IL-22 ameliorates ethanol-induced liver injury [18]. STAT3 activation may also have opposing functions in the liver. While hepatocyte-specific STAT3 knockout alleviates liver inflammation in acute liver injury induced by carbon tetrachloride [32] or ethanol ingestion [33], it enhances liver inflammation in Con A-induced $\mathrm{T}$ cell hepatitis [34] or in LPSinduced liver injury [35], suggesting that hepatic STAT3 activation may serve as either a proinflammatory or an anti-inflammatory signal according to the models. The findings in our study indicated that MSCs- or TSG6-induced suppression of hepatic STAT3 activation might play a beneficial role in relieving liver injury, liver inflammation, and hepatic steatosis and in modulating the inflammatory responses (Figs. 3, 4, and 5). Furthermore, we observed enhanced 


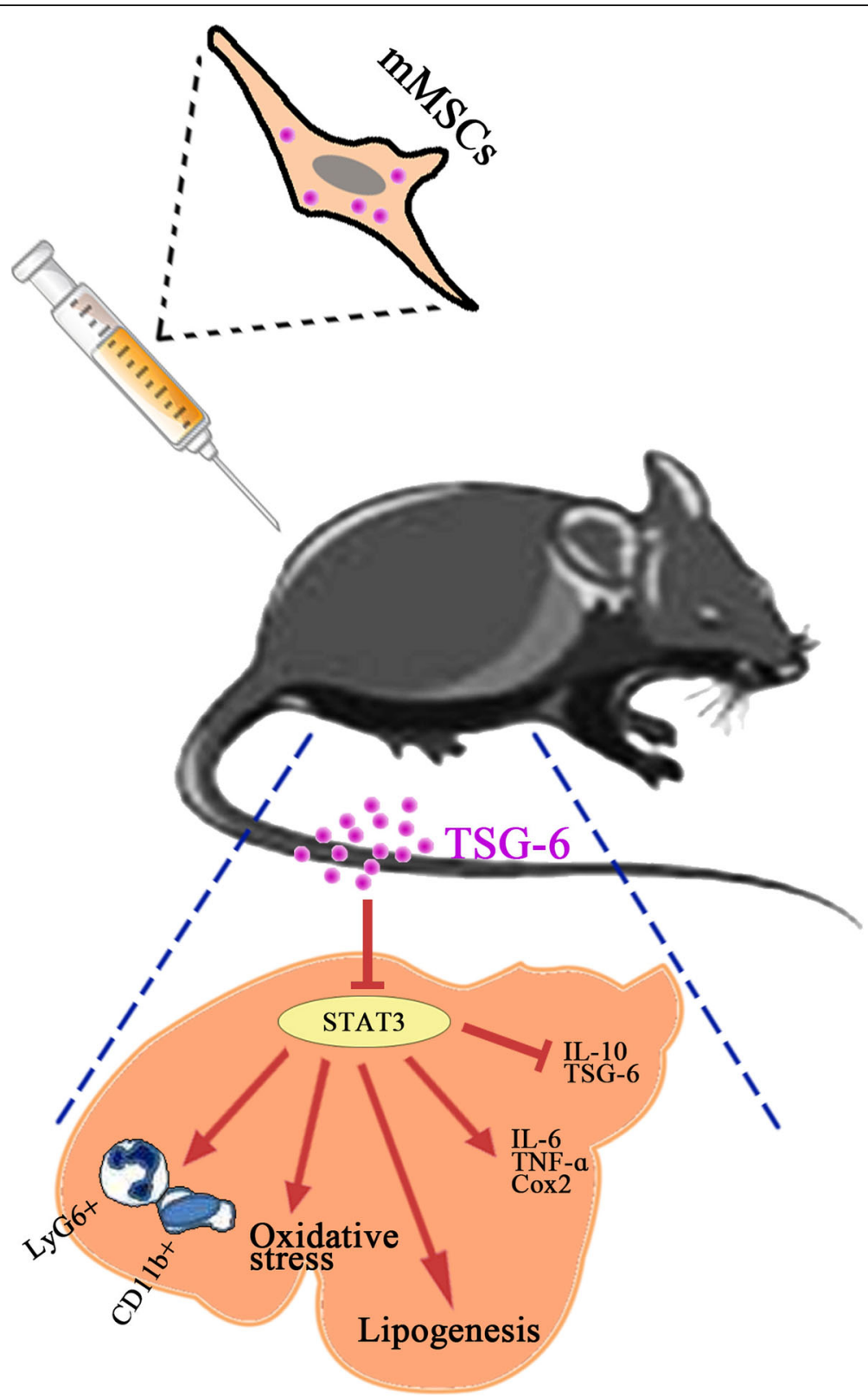

Fig. 7 Schematic overview of mouse MSC activities in the treatment of alcoholic hepatitis

efficacy of MSC therapy in alleviating AH after adding AG490, an inhibitor for STAT3 signaling, to MSC therapy (Additional file 8: Figure S7a-h). This finding provided direct evidence that suppression of hepatic STAT3 signaling by MSCs through TSG- 6 may be the underlying mechanism of its efficacy for $\mathrm{AH}$ in mice (Fig. 6).

There are several limitations to the present study. First, we did not provide sufficient evidence supporting the direct linkage between suppression of hepatic STAT3 activation by MSCs through TSG-6 and improvement of liver injury, hepatic steatosis, oxidative stress, neutrophil and monocyte/macrophage infiltration, and change of serum and hepatic cytokines. Second, we did not identify the cellular source responsible for STAT3 activation, as it may be liver parenchymal cells, hepatic neutrophils, or macrophages. However, our study is the first one that investigates the efficacy of MSCs on AH in mice, and we have five important findings as presented before that may be helpful in developing new therapies for more severe $\mathrm{AH}$.

\section{Conclusion}

In conclusion, our study clearly demonstrated that $\mathrm{BM}-\mathrm{MS}$ were effective in treating $\mathrm{AH}$, and they 
exhibited hepatoprotective, anti-steatogenic, antioxidative, anti-inflammatory, and immunomodulatory effects through secreting TSG-6 and inhibiting hepatic STAT3 signaling. Based on previous studies [19, 20, 26-28] and our findings, we propose that MSCs secreting TSG-6 to suppress hepatic STAT3 signaling may be one underlying mechanism of the efficacy of MSCs for AH in mice (Fig. 7). These findings may help in the development of stem cell therapies for the treatment of ALD.

\section{Supplementary information}

Supplementary information accompanies this paper at https://doi.org/10. 1186/s13287-019-1547-8.

Additional file 1:. Supplementary materials. Mouse bone marrowderived mesenchymal stem cells: isolation, culture, characterization and transfection; sample collection, storage and various assay.

Additional file 2: Figure S1a-I. The primary adherent monolayer cells derived from bone marrow displayed a spindle-shape similar to fibroblasts after culture (a-e); Flow cytometric analysis of MSCs derived from mouse bone marrow at passage three $(\mathrm{F}-\mathrm{l})$; Body weight change and liver/body ratio of mice $(j-1)$. Bars $=100 \mu \mathrm{m}$, seven mice per group were used. ${ }^{*} P<0.05,{ }^{* *} P<0.01,{ }^{* * *} P<0.001$

Additional file 3: Figure S2 a-d. Adipogenic $(a, b)$ and osteogenic $(c, d)$ differentiations of bone marrow-derived MSCs before and after staining. Bars $=100 \mu \mathrm{m}$.

Additional file 4: Figure S3 a-f. Normal (a) and transfected BM-MSCS by scrambled siRNAs (b) or TSG-6 siRNAs (c) as confirmed by RT-qPCR (df). Bars $=100 \mu \mathrm{m}$.

Additional file 5: Figure S4a-I. Flow cytometric analysis of hepatic infiltration by neutrophils with anti-Ly6G antibodies conjugated with fluorescein isothiocyanate (FITC). LyG6+ cells from the control (a), AH $(b, g), A H+M S C s(c, h), A H+M S C s+A G 490$ (d), AH+sc-MSCs (i), AH+siTSG-6MSCs (j), and AH+rmTSG-6 (k) groups and analytic results (e,l); MPO activity(f). Five mice per group were used. ${ }^{*} P<0.05,{ }^{* *} P<0.01,{ }^{* * *} P<0.001$.

Additional file 6: Figure S5a-k. Flow cytometric analysis of hepatic infiltration by monocytes with anti-CD11b antibodies conjugated with allophycocyanin (APC). CD11b+ cells from the control (a), AH (b,f), AH+MSCs (c,g), AH+MSCs+AG490 (d), AH+sc-MSCs (h), AH+siTSG-6-MSCs (i), and AH+rmTSG-6 (j) groups and analytic results (e,k). Five mice per group were used. ${ }^{*} \mathrm{P}<0.05,{ }^{* *} \mathrm{P}<0.01,{ }^{* * *} \mathrm{P}<0.001$.

Additional file 7: Figure S6a-c. Low frequency of SYR + cells were detected in the inflammed liver of mice transplanted with MSCs (a) or transinfected MSCs $(b, c)$. Seven mice per group were used. Bars $=20 \mu \mathrm{m}$.

Additional file 8: Figure S7a-h. Intraperitoneal infusion of AG490 plus MSCs led to more pronounced improvement of liver injury than MSCs alone. ALT and AST levels $(a, b)$, serum TC and TG $(c, d)$, hepatic TC and TG $(e, f)$, hepatic MDA and GSH $(g, h)$. Seven mice per group were used. ${ }^{*} \mathrm{P}<$ $0.05,{ }^{* *} \mathrm{P}<0.01,{ }^{* * *} \mathrm{P}<0.001$

Additional file 9: Tables S1, S2. Primer sequences for quantitative realtime PCR.

\section{Abbreviations}

AH: Alcoholic hepatitis; ALD: Alcoholic liver disease; ALT: Alanine aminotransferase; AST: Aspartate aminotransferase; BM: Bone marrow; Cox: Cyclo-oxygenase; ELISA: Enzyme-linked immunosorbent assay; GFP: Green fluorescent protein; GSH: Glutathione; IL: Interleukin; MAPKs: Mitogen-activated protein kinase; MDA: Malondialdehyde; MPO: Myeloperoxidase; MSCs: Mesenchymal stem cells; NF-kB: Nuclear factor (NF)-кB; NIAAA: The National Institute on Alcohol Abuse and Alcoholism; RTqPCR: Reverse transcription quantitative PCR; SC-MSCs: Scrambled-siRNAMSCS; SD: Standard deviation; siRNA: Small interfering RNA; STAT3: Signal transducer and activator of transcription 3; TC: Total cholesterol; TG: Total triglyceride; TNF: Tumor necrosis factor; TSG-6: TNF-a-stimulated gene/ protein 6

\section{Acknowledgements}

We sincerely thank all the teachers in the Scientific Research Center of Kunming Medical University for their kind and helpful instructions on the use of testing instruments. We sincerely thank Madame Wang (Dr. Yue-Meng Wan's wife) for her kind help in processing the figures.

\section{Authors' contributions}

YMW designed the study, performed the experiments, analyzed the data, and wrote the manuscript; ZQL, QZ, CL, and MJW carried out the animal experiment, MSC isolation and culture, RT-qPCR, ELISA, and WB assays; collected and analyzed the data; and performed the statistical analysis. YHL, ZYX, HMW, YX, JHY, and XFW carried out biochemical tests, MPO activity analysis, liver histology, and immunohistochemistry assays and collected and analyzed the data. XNW designed and supervised the study, performed the statistical analysis, and edited the manuscript. All authors read and approved the final manuscript.

\section{Funding}

This work was supported by the National Natural Science Foundation of China (NSFC) [Grant Numbers 81560525 and 40115048].

\section{Availability of data and materials}

The datasets used and/or analyzed during the current study are available from the corresponding author on reasonable request.

\section{Ethics approval and consent to participate}

All animal experiments were approved by the Ethics Committees of Kunming Medical University (No. KMMU 2019067) and were conducted according to the Guideline of Animal Care and Use Committee of Kunming Medical University.

\section{Consent for publication}

Not applicable.

\section{Competing interests}

The authors declare that they have no competing interests.

\section{Author details}

'Gastroenterology Department, The 2nd Affiliated Hospital of Kunming Medical University, Kunming City 650101, Yunnan Province, China. ${ }^{2}$ Public Health Institute of Kunming Medical University, Kunming City 650500, Yunnan Province, China. ${ }^{3}$ The Biomedical Engineering Research Center, Kunming Medical University, Kunming 650500, Yunnan, China. ${ }^{4}$ Department of Pathology, The 2nd Affiliated Hospital of Kunming Medical University, Kunming City 65010, Yunnan Province, China.

Received: 16 October 2019 Revised: 10 December 2019 Accepted: 29 December 2019 Published online: 13 January 2020

\section{References}

1. Liangpunsakul S, Haber P, McCaughan GW. Alcoholic liver disease in Asia, Europe, and North America. Gastroenterology. 2016;150(8):1786-97.

2. Xiao J, Wang F, Wong NK, He J, Zhang R, Sun R, et al. Global liver disease burdens and research trends: analysis from a Chinese perspective. J Hepatol 2019;71(1):212-21.

3. Gao B, Bataller R. Alcoholic liver disease: pathogenesis and new therapeutic targets. Gastroenterology. 2011;141(5):1572-85.

4. Mathurin P, Beuzin F, Louvet A, Carrié-Ganne N, Balian A, Trinchet JC, et al. Fibrosis progression occurs in a subgroup of heavy drinkers with typical histological features. Aliment Pharmacol Ther. 2007;25(9):1047-54.

5. Lucey MR, Mathurin P, Morgan TR. Alcoholic hepatitis. N Engl J Med. 2009; 360(26):2758-69.

6. Louvet A, Mathurin P. Alcoholic liver disease: mechanisms of injury and targeted treatment. Nat Rev Gastroenterol Hepatol. 2015;12(4):231-42.

7. Lee RH, Pulin AA, Seo MJ, Kota DJ, Ylostalo J, Larson BL, et al. Intravenous hMSCs improve myocardial infarction in mice because cells embolized in lung are activated to secrete the anti-inflammatory protein TSG-6. Cell Stem Cell. 2009;5(1):54-63. 
8. Sala E, Genua M, Petti L, Anselmo A, Arena V, Cibella J, et al. Mesenchymal stem cells reduce colitis in mice via release of TSG6, independently of their localization to the intestine. Gastroenterology. 2015;149(1):163-76 e20.

9. Li Q, Song WJ, Ryu MO, Nam A, An JH, Ahn JO, et al. TSG-6 secreted by human adipose tissue-derived mesenchymal stem cells ameliorates severe acute pancreatitis via ER stress downregulation in mice. Stem Cell Res Ther. 2018;9(1):255

10. Song WJ, Li Q, Ryu MO, Ahn JO, Bhang DH, Jung YC, et al. TSG-6 released from intraperitoneally injected canine adipose tissue-derived mesenchymal stem cells ameliorate inflammatory bowel disease by inducing M2 macrophage switch in mice. Stem Cell Res Ther. 2018;9(1):91.

11. Liu L, Song H, Duan H, Chai J, Yang J, Li X, et al. TSG-6 secreted by human umbilical cord-MSCs attenuates severe burn-induced excessive inflammation via inhibiting activations of P38 and JNK signaling. Sci Rep. 2016;6:30121.

12. Li YH, Xu Y, Wu HM, Yang J, Yang LH, Yue-Meng W. Umbilical cord-derived mesenchymal stem cell transplantation in hepatitis B virus related acute-onchronic liver failure treated with plasma exchange and entecavir: a 24month prospective study. Stem Cell Rev Rep. 2016;12(6):645-53.

13. Cao H, Yang J, Yu J, Pan Q, Li J, Zhou P, et al. Therapeutic potential of transplanted placental mesenchymal stem cells in treating Chinese miniature pigs with acute liver failure. BMC Med. 2012;10:56.

14. Roddy GW, Oh JY, Lee RH, Bartosh TJ, Ylostalo J, Coble K, et al. Action at a distance: systemically administered adult stem/progenitor cells (MSCs) reduce inflammatory damage to the cornea without engraftment and primarily by secretion of TNF-a stimulated gene/protein 6. Stem Cells. 2011; 29(10):1572-9.

15. Choi H, Lee RH, Bazhanov N, Oh JY, Prockop DJ. Anti-inflammatory protein TSG-6 secreted by activated MSCs attenuates zymosan-induced mouse peritonitis by decreasing TLR2/NF-KB signaling in resident macrophages. Blood. 2011;118(2):330-8

16. Lawan A, Bennett AM. Mitogen-activated protein kinase regulation in hepatic metabolism. Trends Endocrinol Metab. 2017;28(12):868-78.

17. Mandrekar P, Szabo G. Signalling pathways in alcohol-induced liver inflammation. J Hepatol. 2009;50(6):1258-66.

18. Ki SH, Park O, Zheng M, Morales-lbanez O, Kolls JK, Bataller R, et al. Interleukin-22 treatment ameliorates alcoholic liver injury in a murine model of chronic-binge ethanol feeding: role of signal transducer and activator of transcription 3. Hepatology. 2010;52(4):1291-300.

19. Norkina O, Dolganiuc A, Catalano D, Kodys K, Mandrekar P, Syed A, et al. Acute alcohol intake induces SOCS1 and SOCS3 and inhibits cytokineinduced STAT1 and STAT3 signaling in human monocytes. Alcohol Clin Exp Res. 2008:32(9):1565-73.

20. Norkina O, Dolganiuc A, Shapiro T, Kodys K, Mandrekar P, Szabo G. Acute alcohol activates STAT3, AP-1, and Sp-1 transcription factors via the family of Src kinases to promote IL-10 production in human monocytes. J Leukoc Biol. 2007:82(3):752-62

21. Bertola A, Park O, Gao B. Chronic plus binge ethanol feeding synergistically induces neutrophil infiltration and liver injury in mice: a critical role for Eselectin. Hepatology. 2013;58(5):1814-23.

22. Wu XQ, Yang Y, Li WX, Cheng YH, Li XF, Huang C, et al. Telomerase reverse transcriptase acts in a feedback loop with NF-KB pathway to regulate macrophage polarization in alcoholic liver disease. Sci Rep. 2016;6:18685.

23. Li HH, Tyburski JB, Wang YW, Strawn S, Moon BH, Kallakury BV, et al. Modulation of fatty acid and bile acid metabolism by peroxisome proliferator-activated receptor a protects against alcoholic liver disease. Alcohol Clin Exp Res. 2014;38(6):1520-31.

24. Leung TM, Nieto N. CYP2E1 and oxidant stress in alcoholic and nonalcoholic fatty liver disease. J Hepatol. 2013;58(2):395-8.

25. Magdaleno F, Blajszczak CC, Nieto N. Key events participating in the pathogenesis of alcoholic liver disease. Biomolecules. 2017;7(1). https://doi org/10.3390/biom7010009.

26. Wang H, Lafdil F, Kong X, Gao B. Signal transducer and activator of transcription 3 in liver diseases: a novel therapeutic target. Int J Biol Sci. 2011;7(5):536-50.

27. Gao B. Cytokines, STATs and liver disease. Cell Mol Immunol. 2005;2:92-100.

28. Fielding CA, McLoughlin RM, McLeod L, Colmont CS, Najdovska M, Grail D, et al. IL-6 regulates neutrophil trafficking during acute inflammation via STAT3. J Immunol. 2008;181(3):2189-95.

29. Yasukawa H, Ohishi M, Mori H, Murakami M, Chinen T, Aki D, et al. IL-6 induces an anti-inflammatory response in the absence of SOCS3 in macrophages. Nat Immunol. 2003;4:551-6.
30. Mandal P, Park PH, McMullen MR, Pratt BT, Nagy LE. The anti-inflammatory effects of adiponectin are mediated via a heme oxygenase-1-dependent pathway in rat Kupffer cells. Hepatology. 2010;51:1420-9.

31. Zhang M, Xu S, Han Y, Cao X. Apoptotic cells attenuate fulminant hepatitis by priming Kupffer cells to produce interleukin-10 through membranebound TGF-beta. Hepatology. 2011;53:306-16.

32. Horiguchi N, Lafdil F, Miller AM, Park O, Wang H, Rajesh M, et al. Dissociation between liver inflammation and hepatocellular damage induced by carbon tetrachloride in myeloid cell-specific signal transducer and activator of transcription 3 gene knockout mice. Hepatology. 2010;51: 1724-34.

33. Horiguchi N, Wang L, Mukhopadhyay P, Park O, Jeong WI, Lafdil F, et al. Cell type-dependent pro- and anti-inflammatory role of signal transducer and activator of transcription 3 in alcoholic liver injury. Gastroenterology. 2008; 134:1148-58.

34. Lafdil F, Wang H, Park O, Zhang W, Moritoki Y, Yin S, et al. Myeloid STAT3 inhibits $T$ cell-mediated hepatitis by regulating $T$ helper 1 cytokine and interleukin-17 production. Gastroenterology. 2009;137(6):2125-35 e1-2.

35. Sakamori R, Takehara T, Ohnishi C, Tatsumi T, Ohkawa K, Takeda K, et al. Signal transducer and activator of transcription 3 signaling within hepatocytes attenuates systemic inflammatory response and lethality in septic mice. Hepatology. 2007:46(5):1564-73.

\section{Publisher's Note}

Springer Nature remains neutral with regard to jurisdictional claims in published maps and institutional affiliations.
Ready to submit your research? Choose BMC and benefit from:

- fast, convenient online submission

- thorough peer review by experienced researchers in your field

- rapid publication on acceptance

- support for research data, including large and complex data types

- gold Open Access which fosters wider collaboration and increased citations

- maximum visibility for your research: over $100 \mathrm{M}$ website views per year

At $\mathrm{BMC}$, research is always in progress.

Learn more biomedcentral.com/submissions 